\title{
Systemic Sclerosis and the Risk of Tuberculosis
}

\author{
Shuo-Ming Ou, Wen-Chien Fan, Kun-Ta Cho, Chiu-Mei Yeh, Vincent Yi-Fong Su, \\ Man-Hsin Hung, Yu-Sheng Chang, Yi-Jung Lee, Yung-Tai Chen, Pei-Wen Chao, \\ Wu-Chang Yang, Tzeng-Ji Chen, Wei-Shu Wang, Hsuan-Ming Tsao, Li-Fu Chen, \\ Fa-Yauh Lee, and Chia-Jen Liu
}

ABSTRACT. Objective. Pulmonary involvement is common in patients with systemic sclerosis (SSc), and this condition causes substantial morbidity and mortality. Disrupted immunity from the disease or associated medication may render such patients subject to tuberculosis (TB) infection. However, the relationship between SSc and TB has not yet been investigated.

Methods. Using the Taiwan National Health Insurance Research Database, 838 patients with SSc diagnosed in Taiwan during 2000-2006 were identified and followed for emergence of TB infection. Incidence rate ratios (IRR) of TB compared to 8380 randomly selected age-, sex-, and comorbidity-matched controls without SSc were calculated. The Cox proportional hazards model was used for multivariate adjustment to identify independent risk factors for TB infection.

Results. The risk of TB infection was higher in the SSc cohort than in controls (IRR 2.81, 95\% CI 1.36-5.37; $\mathrm{p}=0.004$ ), particularly for pulmonary TB (IRR 2.53, 95\% CI 1.08-5.30; p = 0.022). Other independent risk factors for TB infection in patients with SSc were age $\geq 60$ years [hazard ratio (HR) $3.52,95 \% \mathrm{CI} 1.10-11.33 ; \mathrm{p}=0.035$ ] and pulmonary hypertension (PH; HR 6.06, 95\% CI 1.59-23.17; $\mathrm{p}=0.008$ ). Mortality did not differ for SSc patients with or without TB.

Conclusion. In this nationwide study, the incidence of TB infection was significantly higher among patients with SSc than in controls without SSc. Special care should be taken in managing patients with SSc who are at high risk for TB, especially those aged $\geq 60$ years or who also have PH. (First Release July 15 2014; J Rheumatol 2014;41:1662-9; doi:10.3899/jrheum.131125)

Key Indexing Terms:

SYSTEMIC SCLEROSIS PULMONARY FIBROSIS

\section{PULMONARY HYPERTENSION TUBERCULOSIS}

From the Division of Nephrology, the Division of Hematology and Oncology, and the Division of Gastroenterology, Department of Medicine, the Department of Neurology, Neurological Institute, the Department of Chest Medicine, and the Department of Family Medicine, Taipei Veterans General Hospital, Taipei; Division of Chest Medicine, Da-Chien General Hospital, Miaoli; School of Medicine, Institute of Clinical Medicine, Institute of Public Health, and Department of Internal Medicine, National Yang-Ming University; Division of Allergy, Immunology, and Rheumatology, Department of Internal Medicine, Shuang Ho Hospital; Department of Medicine, Taipei City Hospital Heping Fuyou Branch; Department of Anesthesiology, Wan Fang Hospital, Taipei Medical University, Taipei; Department of Emergency Medicine, National Yang-Ming University Hospital, Yilan, Taiwan.

Supported by a grant from Taipei Veterans General Hospital (V101D-0012). The study is based in part on data from the National Health Insurance Research Database provided by the Bureau of National Health Insurance, Department of Health and managed by National Health Research Institutes. The interpretation and conclusions contained herein do not represent those of the Bureau of National Health Insurance, Department of Health, or National Health Research Institutes.

S-M. Ou, MD, Division of Nephrology, Department of Medicine, Taipei Veterans General Hospital, and School of Medicine, National Yang-Ming University; W-C. Fan, MD, Division of Chest Medicine, Da-Chien General Hospital, and School of Medicine, National Yang-Ming University; $K-T$. Cho, $M D$, Institute of Clinical Medicine, National Yang-Ming University, and Department of Chest Medicine, Taipei Veterans General Hospital; C-M. Yeh, MS, School of Medicine, National Yang-Ming University, and Department of Family Medicine, Taipei Veterans General Hospital; V.Y-F. Su, MD, Department of Chest Medicine, Taipei Veterans General Hospital; M-H. Hung, MD, Division of Hematology and Oncology, Department of Medicine, Taipei Veterans General Hospital; Y-S. Chang, MD, School of Medicine, National
Yang-Ming University, and Division of Allergy, Immunology, and Rheumatology, Department of Internal Medicine, Shuang Ho Hospital; $Y$-J. Lee, MD, School of Medicine, National Yang-Ming University, and Department of Neurology, Neurological Institute, Taipei Veterans General Hospital; Y-T. Chen, MD, Division of Nephrology, Department of Medicine, Taipei Veterans General Hospital, and Department of Medicine, Taipei City Hospital Heping Fuyou Branch; P-W. Chao, MD, Department of Anesthesiology, Wan Fang Hospital, Taipei Medical University; W-C. Yang, MD, Division of Nephrology, Department of Medicine, Taipei Veterans General Hospital, and School of Medicine, National Yang-Ming University; T-J. Chen, PhD, Department of Family Medicine, Taipei Veterans General Hospital; W-S. Wang, PhD, School of Medicine, National Yang-Ming University, and Department of Internal Medicine, National Yang-Ming University Hospital; H-M. Tsao, MD, School of Medicine, National Yang-Ming University, and Department of Internal Medicine, National Yang-Ming University Hospital; L-F. Chen, $M D$, Department of Emergency Medicine, National Yang-Ming University Hospital; F-Y. Lee, PhD, School of Medicine, National Yang-Ming University, and Division of Gastroenterology, Department of Medicine, Taipei Veterans General Hospital; C-J. Liu, MD, School of Medicine, National Yang-Ming University, Division of Hematology and Oncology, Department of Medicine, Taipei Veterans General Hospital, Department of Internal Medicine, National Yang-Ming University Hospital, and Institute of Public Health, National Yang-Ming University.

Drs. Shuo-Ming Ou and Wen-Chien Fan contributed equally to this study. Address correspondence to Dr. Chia-Jen Liu, Division of Hematology and Oncology, Department of Medicine, Taipei Veterans General Hospital No. 201, Shipai Road, Sec. 2, Taipei 11217, Taiwan. E-mail: chiajenliu@gmail.com

Accepted for publication April 7, 2014.

Personal non-commercial use only. The Journal of Rheumatology Copyright () 2014. All rights reserved. 
Systemic sclerosis ( $\mathrm{SSc}$ ) is an autoimmune disorder characterized by small vasculopathy, autoantibody production, and fibrosis of multiple organs (skin, gastrointestinal tract, lung, kidney, and heart) ${ }^{1}$. Two major pulmonary involvements associated with SSc, pulmonary hypertension $(\mathrm{PH})$ and interstitial lung disease (ILD), can occur either together or independently. Each pulmonary compromise, when present, is currently the leading cause of morbidity and mortality in patients with $\mathrm{SSc}^{2,3}$. The crucial components underlying the pathogenesis of SSc include endothelial dysfunction and dysregulation of fibroblasts, which results in excessive collagen production, and profound abnormalities of the immune system ${ }^{4}$.

Tuberculosis (TB) remains a major public health problem and is responsible for an estimated 1.7 million global deaths per year ${ }^{5}$. Patients with SSc may be at risk of TB infection because of defective immunity from SSc or associated immunosuppressant therapy. TB might be acquired from an active infected person or from reactivation of a preexisting quiescent lesion. Pulmonary involvement is common in SSc and usually affects the architecture of both the lung tissue and pulmonary vessels ${ }^{6}$. Previous studies demonstrated that structurally damaging lung diseases, such as chronic $\mathrm{ILD}^{7}$, chronic obstructive pulmonary disease $(\mathrm{COPD})^{8}$, bronchiectasis $^{9}$, or pneumoconiosis ${ }^{10}$, can impair local host immunity and further increase susceptibility to TB infection. However, although a few case reports have been published ${ }^{11,12}$, no large-scale study has been conducted on the incidence, risk factors, and mortality rates among patients with SSc who develop TB, to our knowledge. Therefore, we performed a longitudinal nationwide cohort study using the Taiwan National Health Insurance Research Database (NHIRD) to investigate the relationship between SSc and incident TB infection.

\section{MATERIALS AND METHODS}

Data sources. The Taiwanese government began the National Health Insurance (NHI) program in 1995 to provide comprehensive healthcare for all citizens. Enrollment in this program is mandatory, and coverage of the population reached $98.29 \%$ in $2006^{13}$. Comprehensive medical care including outpatient, inpatient, emergency, dental, traditional Chinese medicine services, and prescription drugs are provided by the NHI program. In 1999, the Bureau of National Health Insurance began releasing patient data (e.g., NHI enrollment files, claims data, and a prescription drug registry) in electronic form under the NHIRD project for research.

For catastrophic illnesses such as SSc, the government registered the confirmed subjects, after strict verification ${ }^{14}$, in the special category "Catastrophic Illness" in NHIRD. We obtained data from this dataset that included all claims information for patients with catastrophic illnesses. The application of a catastrophic illness certificate for SSc requires a comprehensive review of medical records, examination reports, and the results of imaging studies by physicians. After successful certification, patients with SSc can be exempted from related medical expenses. Our study was approved by the Institutional Review Board of Taipei Veterans General Hospital (201204022BC).

Study population. We used discharge codes (International Classification of Diseases, 9th revision, and clinical modification ICD-9-CM) in the Registry of Catastrophic Illness. We identified 937 patients who were diagnosed with SSc between January 1, 2000, and December 31, 2006. We defined newly diagnosed TB as a compatible ICD-9-CM code (010-018) plus a prescription for at least 2 anti-TB medications for more than 28 days ${ }^{15,16}$. We further validated our diagnosis and reclassified the patients as non-TB cases if their initial TB-related ICD-9 codes (010-018) in the NHI reimbursement database were changed to other ICD-9 codes during the followup period, including non-TB mycobacterial infection (ICD-9 code 031), lung cancer (ICD-9 code 162), or positive tuberculin skin test (ICD-9 code 795.5), with discontinuation of anti-TB medications ${ }^{17,18}$. To assess the reliability of our findings, we conducted a secondary analysis using a definition of TB diagnosis that required the prescription for at least 3 anti-TB medications.

Patients were excluded who were under 20 years of age, had antecedent $\mathrm{TB}$, were followed for less than 30 days, and/or developed TB within 30 days of diagnosis. A total of 838 patients with SSc were included. We also collected information on comorbidities, including diabetes mellitus, hypertension, COPD, chronic kidney disease, cirrhosis, malignancies, and substance abuse. We also analyzed sociodemographic characteristics, such as age, sex, income (NT $<\$ 20,000$, NT \$20,000-39,999, and NT $\geq$ $\$ 40,000$ ), and level of urbanization (level 1, level 2, and level 3) in our analysis ${ }^{19}$. Urbanization levels are divided according to the Taiwan National Health Research Institute publications. Level 1 designates the most urbanized areas and level 3 designates the least urbanized areas. Medications, including corticosteroids and immunosuppressants, were also retrieved and converted to defined daily dose (DDD), a unit recommended by the World Health Organization. DDD is the assumed average maintenance dose per day of a drug consumed for its main indication in adults and is commonly used for research comparing the use of immunosuppressant agents between international settings ${ }^{20,21}$. Using the concept of DDD, we could compare any immunosuppressant drugs based on the same standard: (total amount of drug)/(amount of drug in a DDD) = number of DDD. Cumulative DDD (cDDD), which indicates exposed duration, was calculated as the sum of dispensed DDD of any immunosuppressant agent. This calculation allowed us to compare use with the risk of subsequent TB. The cDDD of an immunosuppressant agent within 1 year before TB diagnosis was categorized into 2 groups ( $<28$ and $\geq 28 \mathrm{cDDD})$. Drugs used for $<28 \mathrm{cDDD}$ were defined as non-used ${ }^{20}$.

Control cohort. Subjects without SSc were used as a matched cohort and were randomly selected from 1 million NHI beneficiaries out of a population of 21.4 million enrollees throughout Taiwan in 2000. Because $\mathrm{SSc}$ is a relatively rare disorder and the incidence of TB in patients with $\mathrm{SSc}$ is even lower, we matched each patient with SSc with 10 non-SSc subjects by age, sex, and presence of comorbidities on the same index date of diagnosis. The same exclusion criteria were applied to the control cohort as to patients with SSc. A total of 8380 people served as the matched control cohort.

Major outcome measured. The 2 cohorts, SSc cohort and matched cohort, were followed for the emergence of TB infection (ICD-9-CM code 010-018). If no TB occurred, subjects were followed to death, or the end of the study period (2006). Because TB is endemic in Taiwan, the Taiwan Centers for Disease Control (CDC) has declared TB as a mandatory notifiable disease ${ }^{22}$. By law, physicians diagnosing incident TB cases or prescribing anti-TB drugs must report cases to the Taiwan $\mathrm{CDC}$ within 1 week, allowing all newly-diagnosed TB cases to be identified.

Statistical analyses. Extraction and computation of data were done using the Perl programming language (version 5.12.2). Microsoft SQL Server 2005 (Microsoft Corporation) was used for data linkage, processing, and sampling. All statistical analyses were done using IBM SPSS statistical software (version 20.0, IBM-SPSS). Data are expressed as means \pm SD or medians (interquartile ranges) when appropriate. A chi-square test or Fisher's exact test was used for categorical variables, and the Mann-Whitney $U$ test was used for parametric and nonparametric variables. Incidence rates (per 10,000 person-yrs) and incidence rate ratios

Personal non-commercial use only. The Journal of Rheumatology Copyright (c) 2014. All rights reserved. 
(IRR) were analyzed. The Kaplan-Meier method was used for estimation of cumulative incidence. For multivariate adjustment, the Cox proportional hazards model was used to compute hazard ratios (HR) as well as 95\% CI. A $\mathrm{p}<0.05$ was considered significant.

\section{RESULTS}

Patient characteristics. Figure 1 shows the study entry flow chart. A total of 838 patients were included after excluding patients who were younger than age 20 when diagnosed ( $n=40)$, had antecedent TB $(n=44)$, were followed for fewer than 30 days $(n=11)$, or were diagnosed with TB within 30 days after SSc diagnosis $(n=4)$. The study population had a median age of 49 years (range 20-87 yrs), and was $76.3 \%$ women. The baseline characteristics of patients with and without SSc are in Table 1. Age, sex, and underlying comorbidities were matched between the study and control groups.

Comparison of incidence rates of TB between SSc cohort and controls cohort. Kaplan-Meier analysis showed that the cumulative incidence of TB was significantly higher in the SSc cohort than in the control cohort (log-rank test; $\mathrm{p}=$ 0.001; Figure 2). As shown in Table 2, among the 9218 patients (838 SSc cases and 8380 matched controls) in our study during a 7-year period, $60(0.7 \%)$ cases of newly diagnosed TB were identified, including 12 with SSc and 48 in the matched controls. The incidence rate of TB in the $\mathrm{SSc}$ cohort was higher than in the matched cohort (41.4 vs 14.7 per 10,000 person-yrs, $p=0.004)$. The SSc cohort had a 2.81-fold greater risk of TB infection than the matched cohort (IRR 2.81, 95\% CI 1.36-5.37; $\mathrm{p}=0.004$ ). If TB cases were further classified as pulmonary versus extrapulmonary TB, the SSc cohort still had an increased risk of developing pulmonary TB (IRR 2.53, 95\% CI 1.08-5.30; p = 0.022) compared to the matched cohort. Although not significant, the SSc cohort also had an increased risk of developing extrapulmonary TB (IRR 4.22, 95\% CI 0.27-17.57; p = $0.064)$.

As shown in Table 3, we performed multivariable Cox proportional hazards model analysis and found that SSc itself was an independent risk factor for TB infection (HR $2.99,95 \%$ CI $1.58-5.63 ; \mathrm{p}=0.001)$. Other independent risk factors included age $\geq 60$ years (HR $2.79,95 \%$ CI $1.66-4.71 ; \mathrm{p}<0.001)$ and having COPD (HR 2.19, 95\% CI $1.30-3.68 ; \mathrm{p}=0.003)$. We further conducted a secondary

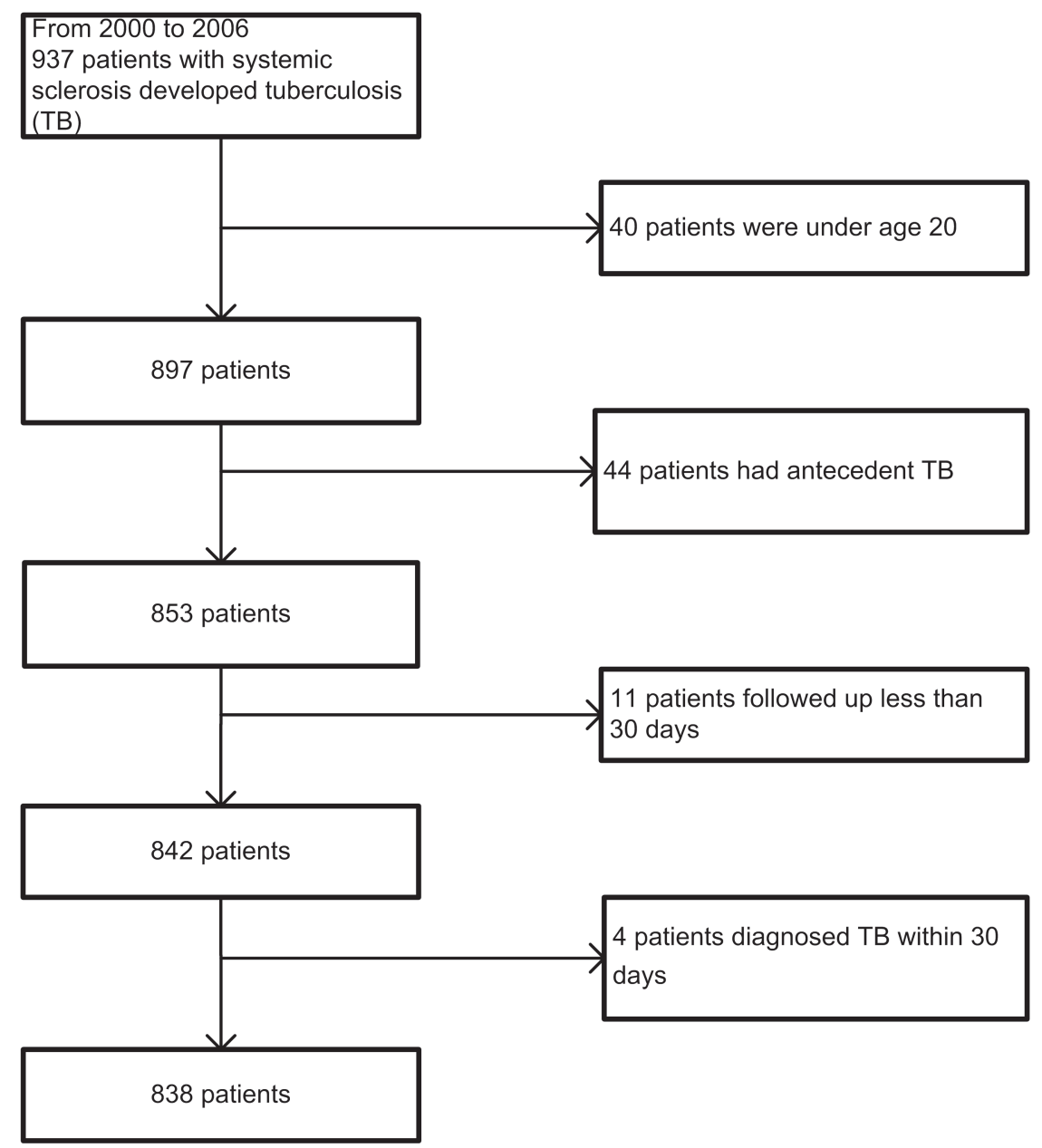

Figure 1. Patient selection flow chart. 
Table 1. Baseline characteristics of patients with systemic sclerosis (SSc) and the matched cohort.

\begin{tabular}{|c|c|c|c|c|c|}
\hline Characteristics & \multicolumn{2}{|c|}{ Patients with SSc, $\mathrm{n}=838$} & \multicolumn{2}{|c|}{ Matched Cohort, $n=8380$} & $\mathrm{p}$ \\
\hline Median age, yrs (range) & \multicolumn{2}{|c|}{$49(20-87)$} & \multicolumn{2}{|c|}{$49(20-87)$} & $1.000^{\mathrm{a}}$ \\
\hline Age, yrs & & & & & \\
\hline$<60$ & 604 & $(72.1)$ & 6040 & $(72.1)$ & $1.000^{\mathrm{b}}$ \\
\hline$\geq 60$ & 234 & (27.9) & 2340 & (27.9) & \\
\hline \multicolumn{6}{|l|}{ Sex } \\
\hline Female & 639 & $(76.3)$ & 6390 & $(76.3)$ & $1.000^{\mathrm{b}}$ \\
\hline Male & 199 & (23.7) & 1990 & (23.7) & \\
\hline \multicolumn{6}{|l|}{ Comorbidity } \\
\hline Diabetes mellitus & 130 & $(15.5)$ & 1297 & $(15.5)$ & $0.978^{b}$ \\
\hline Hypertension & 241 & (28.8) & 2407 & (28.7) & $0.983^{b}$ \\
\hline COPD & 206 & $(24.6)$ & 2068 & $(24.7)$ & $0.951^{\mathrm{b}}$ \\
\hline Chronic kidney disease & 148 & $(17.7)$ & 1483 & $(17.7)$ & $0.979^{\mathrm{b}}$ \\
\hline Liver cirrhosis & 27 & $(3.2)$ & 272 & $(3.2)$ & $0.970^{\mathrm{b}}$ \\
\hline Malignancies & 15 & $(1.8)$ & 153 & (1.8) & $0.941^{\mathrm{b}}$ \\
\hline Substance abuse & 3 & $(0.4)$ & 30 & $(0.4)$ & $1.000^{\mathrm{c}}$ \\
\hline
\end{tabular}

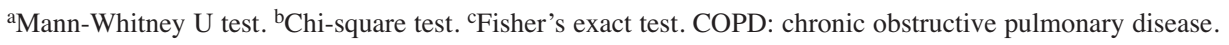

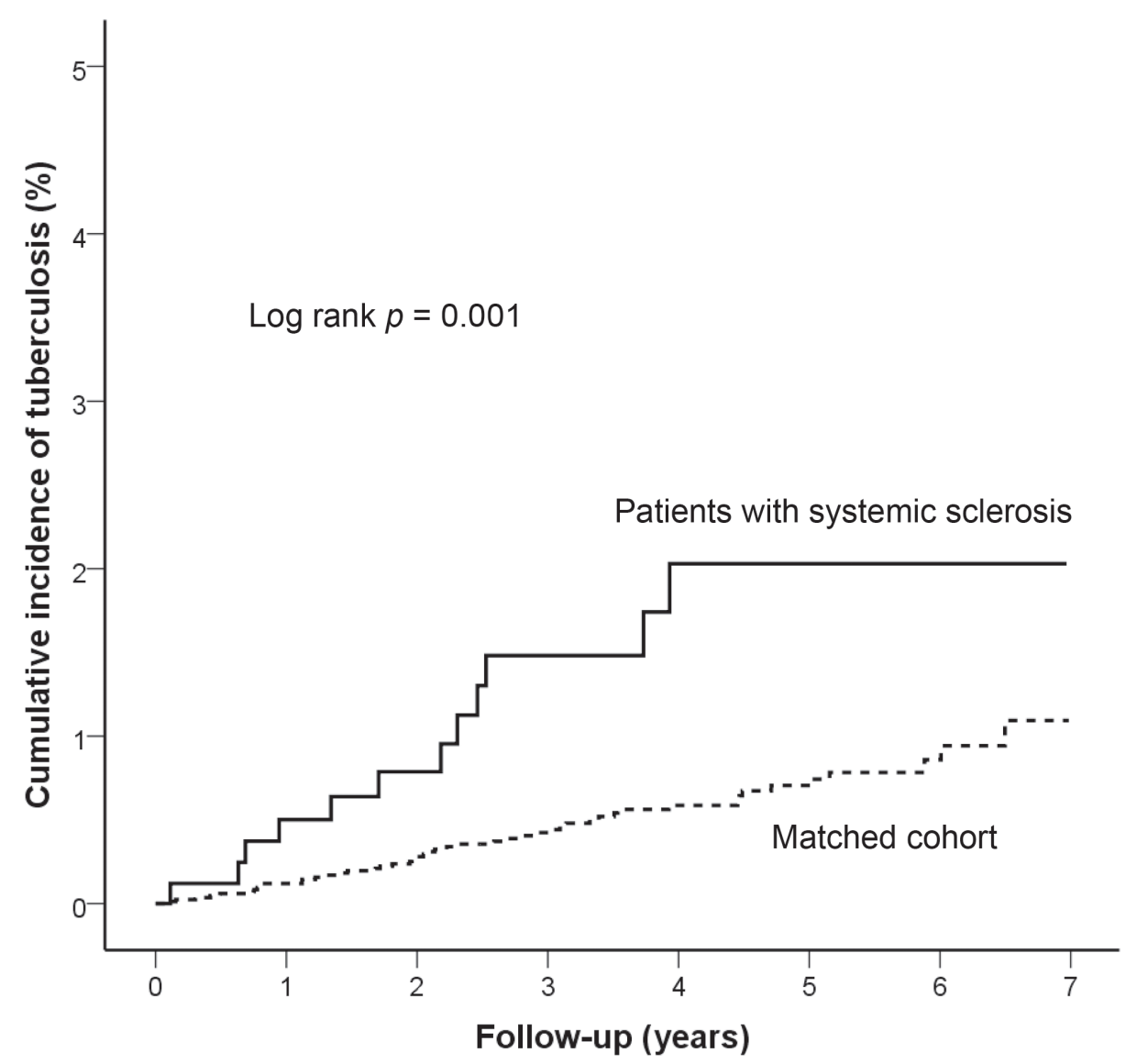

Figure 2. Cumulative incidence of tuberculosis (TB) in patients with systemic sclerosis and the matched cohort. 
Table 2. Incidence of pulmonary and extrapulmonary tuberculosis (TB) in patients with systemic sclerosis (SSc) and the matched cohort.

\begin{tabular}{|c|c|c|c|c|c|c|}
\hline Total & 12 & 41.4 & 48 & 14.7 & $2.81(1.36-5.37)$ & 0.004 \\
\hline Extrapulmonary TB & 3 & 10.3 & 8 & 2.5 & $4.22(0.27-17.57)$ & 0.064 \\
\hline
\end{tabular}

IRR: incidence rate ratio.

Table 3. Cox regression of risk factors for tuberculosis (TB).

\begin{tabular}{lcccc}
\hline \multirow{2}{*}{ Variables } & \multicolumn{2}{c}{ Univariate Analysis } & \multicolumn{2}{c}{ Multivariable Analysis ${ }^{\mathrm{a}}$} \\
& HR $(95 \% \mathrm{CI})$ & $\mathrm{p}$ & $\mathrm{H}(95 \% \mathrm{CI})$ & $\mathrm{p}$ \\
\hline Systemic sclerosis & $2.82(1.50-5.30)$ & 0.001 & $2.99(1.58-5.63)$ & 0.001 \\
Age $\geq 60$ yrs & $3.23(1.94-5.37)$ & $<0.001$ & $2.79(1.66-4.71)$ & $<0.001$ \\
Male sex & $1.74(1.02-2.97)$ & 0.044 & \\
No. outpatient visits & & & \\
$0-5$ & 1 & & \\
$6-10$ & $0.37(0.03-4.09)$ & 0.418 & \\
$>10$ & $0.74(0.18-3.04)$ & 0.673 & \\
Income, NT\$ & & & \\
$\quad<20,000$ & 1 & & \\
$20,000-39,999$ & $0.77(0.44-1.37)$ & 0.374 & \\
$\geq 40,000$ & $0.89(0.45-1.76)$ & 0.735 & \\
Urbanization & & & \\
Level 1 & 1 & & \\
Level 2 & $0.78(0.42-1.45)$ & 0.426 & \\
Level 3 & $1.97(1.00-3.87)$ & 0.050 & \\
Diabetes mellitus & $1.57(0.85-2.90)$ & 0.152 & \\
Hypertension & $1.62(0.96-2.72)$ & 0.070 & \\
COPD & $2.69(1.62-4.47)$ & $<0.001$ & $2.19(1.30-3.68)$ \\
Chronic kidney disease & $1.74(0.98-3.09)$ & 0.058 & \\
\hline
\end{tabular}

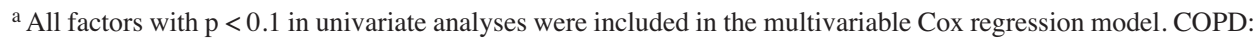
chronic obstructive pulmonary disease; HR: hazard ratio.

analysis using a definition of TB diagnosis requiring prescriptions for at least 3 anti-TB medications, which did not significantly alter the results of the primary analysis (Appendix 1).

$T B$ risk factors in patients with SSc. Of 838 patients with SSc, $521(62.2 \%)$ received corticosteroid, 102 (12.2\%) received cyclophosphamide (CYC), 10 (1.2\%) received cyclosporine, $32(3.8 \%)$ received methotrexate, and 46 (5.5\%) received azathioprine. Univariate Cox regression analysis identified 4 risk factors for TB in patients with SSc: age $\geq 60$, PH, use of corticosteroids, and use of CYC (Table 4). Multivariate Cox regression hazards analysis indicated that 2 variables were independent risk factors for TB in patients with SSc: age $\geq 60$ (HR 3.52, 95\% CI 1.10-11.33; $\mathrm{p}=0.035)$ and having PH (HR 6.06, 95\% CI 1.59-23.17; $p=0.008)$. Other factors, including use of corticosteroids or CYC, were not significant in the multivariable analysis. Of the patients with SSc during the study period, 4 of 12 patients $(33.3 \%)$ with TB died, compared to 119 of 826 patients $(14.4 \%)$ without TB $(\mathrm{p}=0.066)$.

\section{DISCUSSION}

This is the first study, to our knowledge, to investigate the association between $\mathrm{SSc}$ and $\mathrm{TB}$ using a nationwide population-based cohort. We compared the cumulative incidence of TB in patients with SSc to the matched cohort. This study had a long followup period and its findings provide several new insights: (1) patients with SSc had a significantly increased risk of developing TB (IRR 2.81) compared to the matched controls during the 7-year study period; (2) SSc itself might be an independent risk factor for TB development; (3) patients with SSc had 2.53 times greater risk of developing pulmonary TB than patients in the matched cohort; and (4) age $\geq 60$ years and $\mathrm{PH}$ were risk factors for TB in the SSc cohort.

In 1987, Cowie ${ }^{23}$ reported a $40 \%$ incidence of prior TB in black gold miners with SSc. Despite noting a possible association between TB and SSc, the results of that earlier study might be confounded by silica exposure/silicosis. To date, previous studies that have explored the association between SSc and TB are a report of a Japanese-Canadian 
Table 4. Cox regression of risk factors for tuberculosis (TB) in the systemic sclerosis (SSc) cohort.

\begin{tabular}{lcccc}
\hline Variables & \multicolumn{2}{c}{ Univariate Analysis } & \multicolumn{2}{c}{ Multivariable Analysis ${ }^{\mathrm{a}}$} \\
& HR $(95 \% \mathrm{CI})$ & $\mathrm{p}$ & $\mathrm{HR}(95 \% \mathrm{CI})$ & $\mathrm{p}$ \\
\hline Age $\geq 60$ yrs & $4.13(1.31-13.02)$ & 0.016 & $3.52(1.10-11.33)$ & 0.035 \\
Male sex & $1.21(0.33-4.48)$ & 0.775 & & \\
Comorbidity & & & & \\
$\quad$ Hypertension & $2.06(0.66-6.51)$ & 0.216 & & \\
$\quad$ COPD & $2.44(0.77-7.68)$ & 0.129 & & \\
$\quad$ Chronic kidney disease & $0.96(0.21-4.38)$ & 0.957 & & \\
SSc with pulmonary involvement & & & & \\
$\quad$ Pulmonary hypertension & $9.65(2.58-36.11)$ & 0.001 & $6.06(1.59-23.17)$ & \\
$\quad$ Interstitial lung disease & $3.02(0.82-11.17)$ & 0.097 & & \\
Medications, $\geq 28$ cDDD & & & & \\
$\quad$ Corticosteroid & $7.94(1.02-61.50)$ & 0.047 & $7.07(0.91-55.12)$ & \\
$\quad$ Cyclophosphamide & $4.23(1.27-14.06)$ & 0.019 & $2.63(0.78-8.87)$ & \\
$\quad$ Cyclosporine & $0.05(0.00-6.372 \mathrm{E} 8)$ & 0.800 & & \\
$\quad$ Methotrexate & $2.18(0.28-16.90)$ & 0.456 & & \\
$\quad$ Azathioprine & $3.42(0.75-15.60)$ & 0.113 & & \\
\hline
\end{tabular}

${ }^{\text {a }}$ All factors with $\mathrm{p}<0.1$ in univariate analyses were included in the multivariable Cox regression. cDDD: cumulative defined daily dose; HR: hazard ratio; COPD: chronic obstructive pulmonary disease.

woman $^{24}, 2$ Russian cases ${ }^{25,26}$, and an Indian case series of 13 patients with SSc who developed $\mathrm{TB}^{11}$. However, the findings of the Indian report were limited by a small sample size, a lack of a control group, and a high women-to-men ratio (23:1). Our study found a 2.81-fold higher incidence of TB in patients with SSc (41.4 per 10,000 person-yrs) than in matched controls (14.7 per 10,000 person-yrs). Moreover, our study demonstrated that SSc itself was an independent risk factor for $\mathrm{TB}$ infection after adjusting for other confounders.

SSc is an autoimmune disorder characterized by abnormalities of both the cellular and humoral immunity ${ }^{27}$. SSc patients have reduced numbers of circulating T lymphocytes, and reportedly decreased lymphocyte proliferation ${ }^{28,29}$. The subpopulation of $\mathrm{T}$ lymphocytes regulating cell-mediated immunity is also selectively reduced ${ }^{30}$. Impaired immunity could predispose patients with SSc to TB infection.

Our study found that compared to controls, patients with SSc were at a greater risk for developing pulmonary TB. The risk for extrapulmonary TB showed a trend toward significance (IRR $=4.22, \mathrm{p}=0.064)$. Up to $70 \%$ to $90 \%$ of patients with SSc had pulmonary involvement, which might predispose patients to pulmonary $\mathrm{TB}^{31,32}$. To our knowledge, this is the first study to demonstrate that SSc with pulmonary involvement, especially $\mathrm{PH}$, was an independent risk factor for TB (HR 6.06). PH in SSc can result from fibroblast stimulation by a number of cytokines produced by activated macrophages including transforming growth factor (TGF)- $\beta$, tumor necrosis factor (TNF)- $\alpha$, platelet-derived growth factor, and fibronectin. Fibroblast stimulation also leads to increased vascular endothelial growth factor and thus aberrant neoangiogenesis ${ }^{33}$. Increased levels of TNF- $\alpha$ and TGF- $\beta$ are reported to suppress the protective immune response against $\mathrm{TB}^{34,35,36}$. However, work is still needed to elucidate the mechanism of the close relationship between SSc and TB. Further, patients with SSc and ILD had a 3.02 times greater risk for TB, although these results were not significant $(\mathrm{p}=0.097)$. Previous studies found evidence of ILD in $60 \%$ to $91 \%$ of patients with SSc, as evaluated by high-resolution computed tomography. However, only $26 \%$ of the patients were evaluated by physical examination and only $22 \%$ by chest radiograph ${ }^{37,38}$. A high rate of underdiagnosis of ILD in patients with SSc could be the reason the results were not significant.

We observed a trend of higher extrapulmonary involvement in patients with SSc who developed TB than in the matched cohort (IRR 4.22). These data were not significant, possibly because of the limited number of TB subjects $(\mathrm{p}=0.064)$. Our results are similar to previous findings on patients with other autoimmune disorders, systemic lupus erythematous 39,40 , rheumatoid arthritis treated with biological agents ${ }^{41,42}$, and inflammatory myositis, all of which can lead to TB with greater extrapulmonary spread ${ }^{43}$. This is in part due to intrinsic immune dysregulation, permitting the TB bacilli to activate in organs where it is less often successful. The application of systemic corticosteroids and immunosuppressant agents might increase the risk of TB reactivation and dissemination to extrapulmonary sites. A case series by Ahmad, et al ${ }^{11}$ found an increased incidence of TB among patients with SSc receiving dexamethasone therapy. Although we observed that use of corticosteroids was associated with an elevated HR for TB, the result was not statistically significant in the multivariable model. Perhaps use of corticosteroid is a relatively small TB risk factor in this cohort.

Personal non-commercial use only. The Journal of Rheumatology Copyright () 2014. All rights reserved. 
Our study has several limitations. First, the administrative data in the registry database did not contain information about smoking status, physical activity, body mass index, or laboratory and image data. Additionally, data regarding some clinical characteristics (such as the distinction of localized, limited, or diffuse SSc) were not recorded in the NHI database. Therefore, we could not explore the link between these clinical features and TB in patients with SSc. However, this common limitation has been noted in previous registry studies ${ }^{15,44}$. Besides, we did not use the standard procedure for TB diagnosis (acid-fast stain and culturing). Second, subjects who had antecedent TB were not included in our study. The analyses do not determine whether patients with SSc who developed TB had a recent new infection or reactivation of a remote latent infection. Third, physicians apply for a catastrophic illness certificate of SSc based on whether there is the presence of characteristic cutaneous finding of skin thickening, additional extracutaneous manifestations, capillaroscopic abnormalities, and SSc-related autoantibodies. However, the results of diagnostic tests for SSc were not available in our registry data. Finally, we were unable to evaluate the risk of TB development during childhood or adolescence in patients with SSc because we excluded patients $<20$ years old. TB in childhood or adolescence is different from adult TB. Child/adolescent TB often comes from transmission from the community or school and is more likely to be complicated by extrapulmonary involvement ${ }^{45,46}$.

Patients with SSc had a higher risk for pulmonary TB. Hence, a high index of suspicion and more thorough investigation of suspected TB cases, especially in patients with $\mathrm{SSc}$ and $\mathrm{PH}$, could minimize the delay in TB diagnosis and the potential harm to public health in a TB-endemic country.

\section{REFERENCES}

1. Gabrielli A, Avvedimento EV, Krieg T. Scleroderma. N Engl J Med 2009;360:1989-2003.

2. Wells AU, Steen V, Valentini G. Pulmonary complications: one of the most challenging complications of systemic sclerosis. Rheumatology 2009;48 Suppl 3:iii40-4.

3. Steen VD, Medsger TA. Changes in causes of death in systemic sclerosis, 1972-2002. Ann Rheum Dis 2007;66:940-4.

4. Jimenez SA, Derk CT. Following the molecular pathways toward an understanding of the pathogenesis of systemic sclerosis. Ann Intern Med 2004;140:37-50.

5. World Health Organization. Global tuberculosis control: surveillance, planning, financing. 2008. [Internet. Accessed June 10, 2014.] Available from: www.who.int/tb/publications/global_report/2008/en/index.html

6. Sgonc R, Wick G. Pro- and anti-fibrotic effects of TGF-beta in scleroderma. Rheumatology 2008;47 Suppl 5:v5-7.

7. Shachor Y, Schindler D, Siegal A, Lieberman D, Mikulski Y, Bruderman I. Increased incidence of pulmonary tuberculosis in chronic interstitial lung disease. Thorax 1989;44:151-3.

8. Inghammar M, Ekbom A, Engstrom G, Ljungberg B, Romanus V, Lofdahl CG, et al. COPD and the risk of tuberculosis - a population-based cohort study. PloS One 2010;5:e10138.

9. Jordan TS, Spencer EM, Davies P. Tuberculosis, bronchiectasis and chronic airflow obstruction. Respirology 2010;15:623-8.

10. Cochrane AL. Tuberculosis and coalworkers' pneumoconiosis. Br J Tuberc Dis Chest 1954;48:274-85.

11. Ahmad QM, Shah IH, Nauman Q, Sameem F, Sultan J. Increased incidence of tuberculosis in patients of systemic sclerosis on dexamethasone pulse therapy: a short communication from Kashmir. Indian J Dermatol 2008;53:24-5.

12. Manz B, Noack-Wiemers F, Mittag M, Haustein UF, Nenoff P. Death due to pulmonary tuberculosis in progressive systemic sclerosis. J Eur Acad Dermatol Venereol 2002;16:647-8.

13. Lin HP, Deng CY, Chou P. Diagnosis and treatment delay among pulmonary tuberculosis patients identified using the Taiwan reporting enquiry system, 2002-2006. BMC Public Health 2009;9:55.

14. Liu CJ, Chang YS, Teng CJ, Chen TJ, Ou SM, Tzeng CH, et al. Risk of extrathymic cancer in patients with myasthenia gravis in Taiwan: a nationwide population-based study. Eur J Neurol 2012;19:746-51.

15. Wu CY, Hu HY, Pu CY, Huang N, Shen HC, Li CP, et al. Pulmonary tuberculosis increases the risk of lung cancer: a population-based cohort study. Cancer 2011;117:618-24.

16. Chang YS, Liu CJ, Ou SM, Hu YW, Chen TJ, Lee HT, et al. Tuberculosis infection in primary Sjogren's syndrome: a nationwide population based study. Clin Rheumatol 2014;33:377-83.

17. Lin HH, Ezzati M, Chang HY, Murray M. Association between tobacco smoking and active tuberculosis in Taiwan: prospective cohort study. Am J Respir Crit Care Med 2009;180:475-80.

18. Baker MA, Lin HH, Chang HY, Murray MB. The risk of tuberculosis disease among persons with diabetes mellitus: a prospective cohort study. Clin Infect Dis 2012;54:818-25.

19. Hou SW, Lee YK, Hsu CY, Lee CC, Su YC. Increased risk of acute pancreatitis in patients with chronic hemodialysis: a 4-year follow-up study. PloS One 2013;8:e71801.

20. Tsan YT, Lee CH, Wang JD, Chen PC. Statins and the risk of hepatocellular carcinoma in patients with hepatitis B virus infection. J Clin Oncol 2012;30:623-30.

21. WHO Collaborating Center for Drugs Statistics Methodology: ATC Index with DDDs 2003. Oslo, Norway, WHO, 2003.

22. Ou SM, Liu CJ, Teng CJ, Lin YT, Chang YS, Chiang SC, et al. Impact of pulmonary and extrapulmonary tuberculosis infection in kidney transplantation: a nationwide population-based study in Taiwan. Transpl Infect Dis 2012;14:502-9.

23. Cowie RL. Silica-dust-exposed mine workers with scleroderma (systemic sclerosis). Chest 1987;92:260-2.

24. Roddy J, Holtby S, Seigel S. Scleroderma concurrent with culture proven tuberculosis in a Japanese Canadian patient. Rheumatol 1996;23:2168-70.

25. Tiukhtin NS, Berlova ZD. [Exudative pleurisy with a tuberculous etiology in a patient with systemic scleroderma].[Article in Russian] Probl Tuberk 1979;(3):68-70.

26. Kazak TI, Teriaeva MV, Turintsev BB, Davlinskaia OI. [A case of steroid tuberculosis in systemic scleroderma].[Article in Russian] Probl Tuberk 1996;(2):57-8

27. Haustein UF, Anderegg U. Pathophysiology of scleroderma: an update. J Eur Acad Dermatol Venereol 1998;11:1-8.

28. Baron M, Keystone EC, Gladman DD, Lee P, Poplonski L. Lymphocyte subpopulations and reactivity to mitogens in patients with scleroderma. Clin Exp Immunol 1981;46:70-6.

29. Whiteside TL, Kumagai Y, Roumm AD, Almendinger R, Rodnan GP. Suppressor cell function and T lymphocyte subpopulations in peripheral blood of patients with progressive systemic sclerosis. Arthritis Rheum 1983;26:841-7.

30. Behar SM, Porcelli SA. Mechanisms of autoimmune disease induction. The role of the immune response to microbial pathogens. Arthritis Rheum 1995;38:458-76.

Personal non-commercial use only. The Journal of Rheumatology Copyright () 2014. All rights reserved. 
31. Minai OA, Dweik RA, Arroliga AC. Manifestations of scleroderma pulmonary disease. Clin Chest Med 1998;19:713-31, viii-ix.

32. Gohari Moghadam K, Gharibdoost F, Parastandechehr G, Salehian P. Assessments of pulmonary involvement in patients with systemic sclerosis. Arch Iran Med 2011;14:22-6.

33. Johnson SR, Granton JT. Pulmonary hypertension in systemic sclerosis and systemic lupus erythematosus. Eur Respir Rev 2011;20:277-86

34. Deveci F, Akbulut HH, Turgut T, Muz MH. Changes in serum cytokine levels in active tuberculosis with treatment. Mediators Inflamm 2005;2005:256-62.

35. Boom WH, Canaday DH, Fulton SA, Gehring AJ, Rojas RE, Torres M. Human immunity to M. tuberculosis: T cell subsets and antigen processing. Tuberculosis 2003;83:98-106.

36. Sharma S, Bose M. Role of cytokines in immune response to pulmonary tuberculosis. Asian Pac J Allergy Immunol 2001;19:213-9.

37. Steele R, Hudson M, Lo E, Baron M. Clinical decision rule to predict the presence of interstitial lung disease in systemic sclerosis. Arthritis Care Res 2012;64:519-24.

38. Moore OA, Goh N, Corte T, Rouse H, Hennessy O, Thakkar V, et al. Extent of disease on high-resolution computed tomography lung is a predictor of decline and mortality in systemic sclerosis-related interstitial lung disease. Rheumatology 2013;52:155-60.

39. Hou CL, Tsai YC, Chen LC, Huang JL. Tuberculosis infection in patients with systemic lupus erythematosus: pulmonary and extra-pulmonary infection compared. Clin Rheumatol 2008; 27:557-63.
40. Zhang L, Wang DX, Ma L. [A clinical study of tuberculosis infection in systemic lupus erythematosus].[Article in Chinese] Zhonghua Nei Ke Za Zhi 2008;47:808-10.

41. Silva DG, Silva BD, Junqueira-Kipnis AP, Rabahi MF. [Tuberculosis in rheumatoid arthritis patients: the difficulty in making the diagnosis of latent infection].[Article in Portuguese] J Bras Pneumol 2010;36:243-51.

42. Baronnet L, Barnetche T, Kahn V, Lacoin C, Richez C, Schaeverbeke T. Incidence of tuberculosis in patients with rheumatoid arthritis. A systematic literature review. Joint Bone Spine 2011;78:279-84.

43. Marie I, Menard JF, Hachulla E, Cherin P, Benveniste O, Tiev K, et al. Infectious complications in polymyositis and dermatomyositis: a series of 279 patients. Semin Arthritis Rheum 2011;41:48-60.

44. Li SY, Chen TJ, Chung KW, Tsai LW, Yang WC, Chen JY, et al. Mycobacterium tuberculosis infection of end-stage renal disease patients in Taiwan: a nationwide longitudinal study. Clin Microbiol Infect 2011;17:1646-52.

45. Nemir RL. Perspectives in adolescent tuberculosis: three decades of experience. Pediatrics 1986;78:399-405.

46. Wong KS, Chiu CH, Huang YC, Lin TY. Childhood and adolescent tuberculosis in northern Taiwan: an institutional experience during 1994-1999. Acta Paediatr 2001;90:943-7.

APPENDIX 1. Cox regression of risk factors for tuberculosis (TB).

\begin{tabular}{lcccc}
\hline \multirow{2}{*}{ Variables } & \multicolumn{2}{c}{ Univariate Analysis } & \multicolumn{2}{c}{ Multivariable Analysis ${ }^{\mathrm{a}}$} \\
& HR $(95 \% \mathrm{CI})$ & $\mathrm{p}$ & HR $(95 \% \mathrm{CI})$ & $\mathrm{p}$ \\
\hline Systemic sclerosis & $2.85(1.51-5.38)$ & 0.001 & $3.05(1.61-5.76)$ & $<0.001$ \\
Age $\geq 60$ yrs & $3.50(2.08-5.88)$ & $<0.001$ & $2.87(1.68-4.91)$ & $<0.001$ \\
Male sex & $1.83(1.07-3.15)$ & 0.029 & $1.77(1.02-3.05)$ & 0.041 \\
No. outpatient visits & & & & \\
$0-5$ & 1 & & & \\
6-10 & $0.37(0.03-4.10)$ & 0.419 & & \\
$>10$ & $0.71(0.17-2.93)$ & 0.635 & & \\
Income, NT\$ & 1 & & & \\
$\quad<20,000$ & & & & \\
$20,000-39,999$ & $0.78(0.43-1.40)$ & 0.398 & & \\
$\geq 40,000$ & $0.93(0.47-1.86)$ & 0.840 & & \\
Urbanization & & & & \\
Level 1 & 1 & & & \\
Level 2 & $0.83(0.44-1.54)$ & 0.548 & & \\
Level 3 & $2.09(1.06-4.13)$ & 0.035 & & \\
Diabetes mellitus & $1.48(0.78-2.79)$ & 0.227 & & \\
Hypertension & $1.59(0.94-2.71)$ & 0.085 & & \\
COPD & $2.88(1.72-4.81)$ & $<0.001$ & $2.39(1.41-4.05)$ & \\
Chronic kidney disease & $1.52(0.84-2.78)$ & 0.170 & & \\
\hline
\end{tabular}

${ }^{a}$ All factors with $\mathrm{p}<0.1$ in univariate analyses were included in the multivariable Cox regression model. COPD chronic obstructive pulmonary disease; HR: hazard ratio. 


\section{Correction}

Systemic Sclerosis and the Risk of Tuberculosis

Ou S-M, Fan W-C, Cho K-T, Yeh C-M, Su VY-F, Hung

$\mathrm{M}-\mathrm{H}$, et al. Systemic sclerosis and the risk of tuberculosis.

J Rheumatol 2014;41:1662-9. The first name of the third author was given incorrectly. The correct name is Kun-Ta

Chou. We regret the error.

doi:10.3899/jrheum.131125.C1 